\title{
On the Propagation Criterion of Degree $l$ and Order $k$
}

\author{
Claude Carlet \\ GREYC, Université de Caen \\ and \\ INRIA Projet Codes \\ Domaine de Voluceau, BP 105 \\ 78153 Le Chesnay Cedex \\ FRANCE \\ Claude.Carleteinria.fr \\ http: //ww . info. unicaen.fr/"claude/english.html
}

\begin{abstract}
We determine those Boolean functions on $G F(2)^{n}$ which satisfy the propagation criterion of degree $\ell$ and order $k \geq n-\ell-2$. All of theses functions are quadratic. We design nonquadratic Boolean functions satisfying the criterion $P C(\ell)$ of order $k$ by using the MaioranaMcFarland construction involving nonlinear mappings derived from nonlinear codes.
\end{abstract}

\section{Introduction}

The strict avalanche criterion $S A C$ was introduced in 1985 by $\mathrm{A}$. Webster and $\mathrm{S}$. Tavares [24] for the design of the Boolean functions involved in S-boxes. It is related to their dynamic behaviour (when their input is modified) and has been later generalized by B. Preneel et al. [18] who defined the important propagation criterion.

Let $n$ and $\ell$ be positive integers. A Boolean function $f(x)$, where $x=$ $\left(x_{1}, \cdots, x_{n}\right)$, defined on $G F(2)^{n}$ satisfies the propagation criterion of degree $\ell$, $P C(\ell)$, if for any nonzero word $a \in G F(2)^{n}$ of Hamming weight $\omega(a) \leq \ell$, the Boolean function on $G F(2)^{n}$ equal to $f(x)+f(x+a)$ is balanced (i.e. takes the values 0 and 1 equally often). This criterion is invariant under the permutations and the complementations of coordinates $x_{1}, \cdots, x_{n}$ and the addition to $f$ of any affine function. The strict avalanche criterion is equivalent to $P C(1)$. The notion of perfect nonlinear Boolean function on $G F(2)^{n}$, introduced by W. Meier and $O$. Staffelbach [17], is also generalized by the propagation criterion: the perfect nonlinear functions are those functions which satisfy $P C(\ell)$ for any $\ell$, i.e. those which satisfy $P C(n)$. They exist when $n$ is even, only. In this case, a Boolean function on $G F(2)^{n}$ is perfect nonlinear if and only if it is bent (cf. $[10,20]$ ).

To prevent them from attacks fixing coordinates, we need to consider Boolean functions $f(x)$ which satisfy $P C(\ell)$ when we keep constant a certain number $k$ of coordinates $x_{1}, \cdots, x_{n}$ of $x$. This criterion, also introduced by B. Preneel [19], is called the propagation criterion $P C(\ell)$ of order $k$. Notice that if a function $f$ 
on $G F(2)^{n}$ satisfies the propagation criterion of degree $\ell$ and order $n-\ell$, then it satisfies $P C\left(\ell^{\prime}\right)$ of order $n-\ell$ for every $\ell^{\prime}$ (and $\ell$ must then be even). In sections 2 and 3, we determine those functions which satisfy the propagation criterion of degree $\ell$ and orders greater than or equal to $n-\ell-2$. Notice also that, if a function $f$ satisfies $P C(\ell)$ of order $k \leq n-\ell$, then it satisfies $P C(\ell)$ of order $k^{\prime}$ for any $k^{\prime} \leq k$ : to check this, it is enough to consider the case $k^{\prime}=k-1$; let $f^{\prime}$ be the restriction of $f$ obtained by keeping constant $k-1$ coordinates - say the last $(k-1)$ ones; let $a \in G F(2)^{n-k+1}$ have weight at most $\ell$; since $\ell \leq n-k$, there exists $i \leq n-k+1$ such that $a_{i}=0$; both restrictions of the function $f^{\prime}(x)+f^{\prime}(x+a)$ obtained by keeping constant $x_{i}$ are then balanced, since $f^{\prime}$ satisfies $P C(\ell)$ of order 1 . This implies that the function $f^{\prime}(x)+f^{\prime}(x+a)$ itself is balanced. In particular, $P C(\ell)$ of order $k$ is a stronger condition than $P C(\ell)$ (i.e. $P C(\ell)$ of order 0 ). It is actually more difficult to satisfy.

$\mathrm{K}$. Kurosawa and T. Satoh give in [16] a nice general method to design such functions. But, as we explain it in subsection 4.2, the functions they obtain have a peculiarity which weakens them. Section 4 is devoted to an improvement - via a generalization - of their construction.

\section{Functions satisfying the propagation criterion of degree at least $n-2$}

The characterization of the functions satisfying the propagation criterion of degree $n-2$ will be necessary in the next section. It has been studied by Hirose and Ikeda in a technical report (cf. [13]). We give in proposition 1 the precise description of these functions (which was not given by Hirose and Ikeda).

Proposition 1. Let $n$ be even $\geq 4$. The only Boolean functions on $G F(2)^{n}$ which satisfy $P C(n-2)$ are the perfect nonlinear functions.

Let $n$ be odd $\geq 3$. The Boolean functions satisfying $P C(n-1)$ are the functions of the form:

$$
f\left(x_{1}, \cdots, x_{n}\right)=g\left(x_{1}+x_{n}, \cdots, x_{n-1}+x_{n}\right)+h\left(x_{1}, \cdots, x_{n}\right)
$$

where $g$ is any perfect nonlinear function on $G F(2)^{n-1}$ and $h$ any affine function on $G F(2)^{n}$.

The functions satisfying $P C(n-2)$ are the functions $f\left(x_{1}, \cdots, x_{n}\right)$ of the form (1) and those of the two following forms:

$$
\begin{gathered}
g\left(x_{1}+x_{n}, \cdots, x_{i-1}+x_{n}, x_{i}, x_{2+1}+x_{n}, \cdots, x_{n-1}+x_{n}\right)+h\left(x_{1}, \cdots, x_{n}\right) \\
g\left(x_{1}+x_{n-1}, \cdots, x_{n-2}+x_{n-1}, x_{n}\right)+h\left(x_{1}, \cdots, x_{n}\right)
\end{gathered}
$$

where $g$ and $h$ are as above. Equivalently, for odd $n \geq 3$, the functions satisfying $P C(n-2)$ are those functions $f$ for which:

- there exists a nonzero word a of Hamming weight $\omega(a) \geq n-1$ such that the function $f(x)+f(x+a)$ is constant,

- for every nonzero word $b \neq a$, the function $f(x)+f(x+b)$ is balanced on $G F(2)^{n}$. 
Thus, if a function satisfies $P C(n-2)$, then it admits one linear structure and only one. This linear structure has weight $n-1$ or $n$.

\section{Functions satisfying $P C(\ell)$ of order greater than or equal to $n-\ell-2$}

\subsection{Functions satisfying $P C(\ell)$ of order $n-\ell$}

A function $f$ satisfies $P C(\ell)$ of order $k=n-\ell$ if and only if every restriction of $f$ obtained by keeping constant $n-\ell$ coordinates is perfect nonlinear. Thus, $\ell$ must be even. For $n$ even, these functions were characterized in [6] (cf. proposition 1, page 147, and theorem 1, page 154) and called hyper-bent. We can easily deduce their characterization for any $n$ (and any even $\ell$ ):

Proposition 2. For every $n \geq 4$ and every even $\ell$ such that $2 \leq \ell \leq n-2$, the functions $f$ which satisfy $P C(\ell)$ of order $n-\ell$ are the functions

$$
f\left(x_{1}, \cdots, x_{n}\right)=\sum_{1 \leq i<j \leq n} x_{i} x_{j}+h\left(x_{1}, \cdots, x_{n}\right)
$$

where $h$ is affine.

For every odd $n \geq 3$, the functuons $f$ which satisfy $P C(n-1)$ of order 1 are the functions of the form (1) (cf. the statement of proposition 1).

\subsection{Functions satisfying $P C(\ell)$ of order $n-\ell-1$}

Proposition 3. For every $n \geq 3$ and every postuve even $\ell \leq n-1$, the functions $f$ which satisfy $P C(\ell)$ of order $n-\ell-1$ are the same as those which satisfy $P C(\ell)$ of order $n-\ell$.

For every odd $\ell \geq 3$ and every $n \geq \ell+1$, the functions $f$ whuch satisfy $P C(\ell)$ of order $n-\ell-1$ are the same as those uhich satisfy $P C(\ell+1)$ of order $n-\ell-1$.

\section{Proof:}

Let $f$ satisfy $P C(\ell)$ of order $n-\ell-1$. Assume first that $\ell$ is even.

If $\ell \leq n-2$, then every restriction of $f$ obtained by keeping constant $n-\ell-2$ coordinates is a Boolean function on $G F(2)^{\ell+2}$ which satisfies $P C(\ell)$. Thus, it satisfies $P C(\ell+2)$, according to proposition 1 (applied with $n=\ell+2$ ). Hence, $f$ satisfies $P C(\ell+2)$ of order $n-\ell-2$, and according to proposition 2 , it has the form (4), i.e. satisfies $P C(\ell)$ of order $n-\ell$. The converse is obvious.

If $n$ is odd and $\ell=n-1$, the result holds since, according to the last sentence of proposition 2 , the functions satisfying $P C(n-1)$ are the same as those which satisfy $P C(n-1)$ of order 1 .

Assume now that $\ell$ is odd. Every restriction of $f$ obtained by keeping constant $n-\ell-1$ coordinates is a Boolean function on $G F(2)^{\ell+1}$ and satisfies $P C(\ell)$. Thus, according to proposition 1 (applied with $n=\ell+1$ ) and since $\ell+1 \geq 4$, it satisfies $P C(\ell+1)$ and $f$ satisfies $P C(\ell+1)$ of order $n-\ell-1$. The converse is obvious. 


\subsection{Functions satisfying $P C(\ell)$ of order $n-\ell-2$}

The determination of these functions is more difficult. The key-result is the following:

Proposition 4. For every even $n \geq 8$, every function $f$ satisfying $P C(n-3)$ of order 1 is perfect nonlinear.

\section{Proof:}

Suppose $f$ is not perfect nonlinear, then it does not satisfy $P C(n-2)$, according to proposition 1 . We shall prove that this leads to a contradiction. Since $f$ satisfies $P C(n-3)$ and does not satisfy $P C(n-2)$, there exists a word $a \in G F(2)^{n}$ of weight $n-2$, such that the function $f(x)+f(x+a)$ is not balanced. We may without loss of generality assume that $a=(0,0,1, \cdots, 1)$. Set $a^{\prime}=(0,1, \cdots, 1) \in G F(2)^{n-1}$. Let $f_{0}$ (resp. $\left.f_{1}\right), f_{0}^{\prime}$ (resp. $\left.f_{1}^{\prime}\right)$ be the restrictions of $f$ obtained by keeping $x_{1}$ equal to 0 (resp. 1), $x_{2}$ equal to 0 (resp. 1). The functions $f_{0}, f_{1}, f_{0}^{\prime}$ and $f_{1}^{\prime}$ satisfy $P C(n-3)$, by hypothesis. Thus, according to proposition 1, any of the functions $f_{0}(x)+f_{0}\left(x+a^{\prime}\right), f_{1}(x)+f_{1}\left(x+a^{\prime}\right)$, $f_{0}^{\prime}(x)+f_{0}^{\prime}\left(x+a^{\prime}\right)$ and $f_{1}^{\prime}(x)+f_{1}^{\prime}\left(x+a^{\prime}\right)$ is either constant or balanced. Since the function $f(x)+f(x+a)$ is not balanced, one of the functions $f_{0}(x)+f_{0}\left(x+a^{\prime}\right)$, $f_{1}(x)+f_{1}\left(x+a^{\prime}\right)$ and one of the functions $f_{0}^{\prime}(x)+f_{0}^{\prime}\left(x+a^{\prime}\right), f_{1}^{\prime}(x)+f_{1}^{\prime}\left(x+a^{\prime}\right)$ must be constant. We may without loss of generality assume that these constant functions are $f_{0}(x)+f_{0}\left(x+a^{\prime}\right)$ and $f_{0}^{\prime}(x)+f_{0}^{\prime}\left(x+a^{\prime}\right)$ (otherwise, if for instance they are $f_{1}(x)+f_{1}\left(x+a^{\prime}\right)$ and $f_{0}^{\prime}(x)+f_{0}^{\prime}\left(x+a^{\prime}\right)$, we may change $f\left(x_{1}, \cdots, x_{n}\right)$ into $f\left(x_{1}+1, x_{2}, \cdots, x_{n}\right)$ ). We may also assume that they are equal to 0 (otherwise, they are both equal to 1 and we may change $f$ into $f+1$ ). Let $f_{0,0}^{\prime \prime}$ (resp. $\left.f_{0,1}^{\prime \prime}, f_{1,0}^{\prime \prime}, f_{1,1}^{\prime \prime}\right)$ be the restrictions of $f$ obtained by keeping $\left(x_{1}, x_{2}\right)$ equal to $(0,0)$ (resp. $(0,1),(1,0),(1,1))$. Set $a^{\prime \prime}=(1, \cdots, 1) \in G F(2)^{n-2}$.

We have: $f_{0,0}^{\prime \prime}(x)+f_{0,0}^{\prime \prime}\left(x+a^{\prime \prime}\right)=f_{0,1}^{\prime \prime}(x)+f_{0,1}^{\prime \prime}\left(x+a^{\prime \prime}\right)=f_{1,0}^{\prime \prime}(x)+f_{1,0}^{\prime \prime}\left(x+a^{\prime \prime}\right)=$ 0 . Since the functions $f_{1}^{\prime}(x)+f_{1}\left(x+a^{\prime}\right)$ and $f_{1}^{\prime}(x)+f_{1}^{\prime}\left(x+a^{\prime}\right)$ are either constant or balanced, $f_{1,1}^{\prime \prime}(x)+f_{1,1}^{\prime \prime}\left(x+a^{\prime \prime}\right)$ must be either equal to the constant function 0 or to the constant function 1 . Say $f_{1,1}^{\prime \prime}(x)+f_{1,1}^{\prime \prime}\left(x+a^{\prime \prime}\right)=\epsilon, \epsilon \in G F(2)$. From the equalities $f_{0,0}^{\prime \prime}(x)+f_{0,0}^{\prime \prime}\left(x+a^{\prime \prime}\right)=f_{0,1}^{\prime \prime}(x)+f_{0,1}^{\prime \prime}\left(x+a^{\prime \prime}\right)=f_{1,0}^{\prime \prime}(x)+f_{1,0}^{\prime \prime}\left(x+a^{\prime \prime}\right)=0$ and $f_{1,1}(x)+f_{1,1}^{\prime \prime}\left(x+a^{\prime \prime}\right)=\epsilon$, we deduce:

$$
f(x+a)=f(x)+\epsilon x_{1} x_{2} .
$$

Let $g$ be the restriction of $f$ to the hyperplane of equation $x_{n}=0$. We have, for every $\left(x_{1}, \cdots, x_{n-1}\right) \in G F(2)^{n-1}: f\left(x_{1}, \cdots, x_{n-1}, 1\right)=f\left(x_{1}, x_{2}, x_{3}+1, \cdots, x_{n-1}+\right.$ $1,0)+\epsilon x_{1} x_{2}=g\left(x_{1}, x_{2}, x_{3}+1, \cdots, x_{n-1}+1\right)+\epsilon x_{1} x_{2}$. We deduce:

$$
f\left(x_{1}, \cdots, x_{n}\right)=g\left(x_{1}, x_{2}, x_{3}+x_{n}, \cdots, x_{n-1}+x_{n}\right)+\epsilon x_{1} x_{2} x_{n} .
$$

We show now that the case $\epsilon=0$ is impossible. In this case, we have, for any word $u=\left(u_{1}, \cdots, u_{n}\right)$ :

$$
\begin{array}{r}
f\left(x_{1}, \cdots, x_{n}\right)+f\left(x_{1}+u_{1}, \cdots, x_{n_{2}}+u_{n}\right)=g\left(x_{1}, x_{2}, x_{3}+x_{n}, \cdots, x_{n-1}+x_{n}\right)+ \\
g\left(x_{1}+u_{1}, x_{2}+u_{2}, x_{3}+x_{n}+u_{3}+u_{n}, \cdots, x_{n-1}+x_{n}+u_{n-1}+u_{n}\right) .
\end{array}
$$


Since $f$ satisfies $P C(n-3)$, for every nonzero word $u$ of weight smaller than or equal to $n-3$, the function over $G F(2)^{n-1}$ :

$g\left(x_{1}, x_{2}, x_{3}, \cdots, x_{n-1}\right)+g\left(x_{1}+u_{1}, x_{2}+u_{2}, x_{3}+u_{3}+u_{n}, \cdots, x_{n-1}+u_{n-1}+u_{n}\right)$

is balanced (its weight is half the weight of the function $f\left(x_{1}, \cdots, x_{n}\right)+f\left(x_{1}+\right.$ $u_{1}, \cdots, x_{n}+u_{n}$ ) that is balanced). But, when $u$ ranges over the set of all the nonzero words of length $n$ and weights smaller than or equal to $n-3$, the word $\left(u_{1}, u_{2}, u_{3}+u_{n}, \cdots, u_{n-1}+u_{n}\right)$ ranges over the set of all the nonzero words of length $n-1$ : taking $u_{n}=0$, we obtain all the nonzero words of weights smaller than or equal to $n-3$; and taking $u_{n}=1$, we obtain, among others, all the words of weights greater than or equal to $n-2$ (any such word $v$ is obtained from the word $\left(v_{1}, v_{2}, v_{3}+1, \cdots, v_{n-1}+1,1\right)$ which has weight at most $\left.4 \leq n-3\right)$. Thus, $g$ would be perfect nonlinear, which is impossible since $n-1$ is odd.

We show finally that the case $\epsilon=1$ is also impossible. By hypothesis, the restrictions of $f$ to the hyperplanes of equations $x_{n}=0$ and $x_{3}=0$ both satisfy $P C(n-3)$. The first one is $g$ and the second one is the function:

$$
f\left(x_{1}, x_{2}, 0, x_{4}, \cdots, x_{n}\right)=g\left(x_{1}, x_{2}, x_{n}, x_{4}+x_{n} \cdots, x_{n-1}+x_{n}\right)+x_{1} x_{2} x_{n} .
$$

We deduce that $g$ and the function

$$
r\left(x_{1}, x_{2}, \cdots, x_{n-1}\right)=g\left(x_{1}, x_{2}, x_{3}, x_{4}+x_{3} \cdots, x_{n-1}+x_{3}\right)+x_{1} x_{2} x_{3}
$$

both satisfy $P C(n-3)$. According to proposition $1, g$ admits one linear structure $b$ of weight greater than or equal to $n-2$. Set $c=\left(b_{1}, b_{2}, b_{3}, b_{4}+b_{3}, \cdots, b_{n-1}+b_{3}\right)$. We have, for every $x \in G F(2)^{n-1}$ :

$$
\begin{gathered}
r(x)+r(x+c)=g\left(x_{1}, x_{2}, x_{3}, x_{4}+x_{3} \cdots, x_{n-1}+x_{3}\right)+ \\
g\left(x_{1}+b_{1}, x_{2}+b_{2}, x_{3}+b_{3}, x_{4}+x_{3}+b_{4} \cdots, x_{n-1}+x_{3}+b_{n-1}\right)+ \\
x_{1} x_{2} x_{3}+\left(x_{1}+b_{1}\right)\left(x_{2}+b_{2}\right)\left(x_{3}+b_{3}\right) .
\end{gathered}
$$

But $g\left(x_{1}, x_{2}, x_{3}, x_{4}+x_{3} \cdots, x_{n-1}+x_{3}\right)+g\left(x_{1}+b_{1}, x_{2}+b_{2}, x_{3}+b_{3}, x_{4}+x_{3}+\right.$ $\left.b_{4} \cdots, x_{n-1}+x_{3}+b_{n-1}\right)$ being constant and the functions $x_{1} x_{2} x_{3}$ and $\left(x_{1}+\right.$ $\left.b_{1}\right)\left(x_{2}+b_{2}\right)\left(x_{3}+b_{3}\right)$ having weight $2^{n-4}$ and being different one from each other (since $b$ has weight greater than or equal to $n-2$ ), the function $r(x)+r(x+c)$ can not be constant neither balanced. A contradiction with the fact that $r$ satisfies $P C(n-3)$ and with proposition 1 .

\section{Remark:}

In proposition 4 , the condition $n \geq 8$ can not be weakened: for $n \leq 6$, the functions satisfying $P C(n-3)$ of order 1 are not necessarily perfect nonlinear. For instance, let $r$ be a perfect nonlinear function on $G F(2)^{4}$ and $f$ the function on $G F(2)^{6}$ defined by:

$$
f\left(x_{1}, \cdots, x_{6}\right)=r\left(x_{1}+x_{5}+x_{6}, x_{2}+x_{5}+x_{6}, x_{3}+x_{5}, x_{4}+x_{6}\right) .
$$

$f$ is not perfect nonlinear since it admits the linear structure $(0,0,1,1,1,1)$. It satisfies $P C(n-3)$ of order 1 , since all its restrictions obtained by keeping 
constant one coordinate satisfy $P C(3)$. For instance, keep constant $x_{1}=0$, then the function $f\left(0, x_{1}, \cdots, x_{5}\right)=r\left(x_{4}+x_{5}, x_{1}+x_{4}+x_{5}, x_{2}+x_{4}, x_{3}+x_{5}\right)$ has the form (2) (cf. proposition 1) with $g\left(x_{1}, \cdots, x_{4}\right)=r\left(x_{4}, x_{1}+x_{4}, x_{2}+x_{4}, x_{3}\right)$ (which is a perfect nonlinear function since $r$ is one), $h(x)=0$ and $i=3$.

Theorem 5. For every positive even $\ell \leq n-4(n \geq 6)$ and every odd $\ell$ such that $5 \leq \ell \leq n-5$ ( $n \geq 10$ ), the functions satisfying $P C(\ell)$ of order $n-\ell-2$ are the same as the functions which satisfy $P C(\ell)$ of order $n-\ell$, i.e. they are the functions of the form:

$$
\sum_{1 \leq \imath<j \leq n} x_{\imath} x_{\jmath}+h\left(x_{1}, \cdots, x_{n}\right)
$$

where $h$ is affine.

Proof:

Assume that $\ell$ is even, $\ell \leq n-4$. Every restriction of $f$ obtained by keeping constant $n-\ell-2$ coordinates satisfies $P C(\ell+2)$, according to Proposition 1 (it satisfies $P C(\ell)$ and $\ell+2 \geq 4)$. Thus $f$ satisfies $P C(\ell+2)$ of order $n-\ell-2$ and has form (4).

Assume that $\ell$ is odd. Any restriction of $f$ obtained by keeping constant $n-\ell-3$ coordinates is a Boolean function on $G F(2)^{\ell+3}$ and satisfies $P C(\ell$ of order 1 . According to Proposition 4 and since $\ell+3 \geq 8$, it is perfect nonlinear. Thus, $f$ satisfies $P C(\ell+3)$ of order $n-\ell-3$ and has the form (4).

\section{Construction of nonquadratic functions satisfying $P C(\ell)$ of order $k$}

We have seen above that the functions satisfying $P C(\ell)$ of order $k=n-\ell-2$ are all quadratic for $k \geq 3$ and $n$ sufficiently large. This makes these functions inappropriate for cryptographic use (see for instance [15]). We want now to design nonquadratic functions satisfying $P C(\ell)$ of order $k$ with $3 \leq k<n-\ell-2$.

\subsection{Preliminaries on resilient functions}

A function $f$ from $G F(2)^{n}$ to $G F(2)^{m}$ is $k$-th order correlation-immune if the probability distribution of the values of $f\left(x_{1}, \cdots, x_{n}\right)$, where $x_{1}, \ldots, x_{n}$ are considered as random input variables assuming values from $G F(2)$ with independent equiprobable distributions, is unaltered when at most $k$ of the coordinates $x_{1}, \ldots, x_{n}$ are kept constant. It is $k$-resilient if it is balanced (i.e. takes all of the values in $G F(2)^{m}$ equally often) and $k$-th order correlation-immune.

Remark: $f$ is $k$-resilient if and only if all the sets $f^{-1}(c), c \in G F(2)^{m}$ have the same cardinality and if, for any $c$ in $G F(2)^{m}$, the indicator of $f^{-1}(c)$ (i.e. the Boolean function whose support is $\left.f^{-1}(c)\right)$ is a $k$-th order correlation-immune function. 
We shall use in the sequel the following well-known property (cf. $[1,3,22]$ ):

Property 1: if $f$ is $k$-resilient from $G F(2)^{n}$ to $G F(2)^{m}$ and if $g$ is balanced on $G F(2)^{m}$, then $g \circ f$ is $k$-resilient.

We shall also use a result from Delsarte [8]:

Lemma 6. A Boolean function $f$ on $G F(2)^{n}$ is $k$-th order correlation-immune if and only if, for every nonzero $b \in G F(2)^{n}$ of weight smaller than or equal to $k$, the restriction of the linear form $x \rightarrow b \cdot x$ (where" ." is the usual inner product on $G F(2)^{n}$ ) to the support of $f$ (i.e. the set $\left\{x \in G F(2)^{n} \mid f(x)=1\right\}$ ) is balanced.

\subsection{The Maiorana-McFarland construction}

In [16] is given a construction of $P C(\ell)$ of order $k$ functions which uses a general method for designing Boolean cryptographic functions. This method was first introduced by Maiorana and Mc Farland in the 70's to obtain perfect nonlinear functions (cf. [10]). This construction has been used in several papers (cf. for instance $[5,7])$ to design also resilient functions.

Definition 7. We call Maiorana-McFarland function any Boolean function $f$ defined the following way:

$s, t$ are any positive integers; $n=s+t ; g$ is any Boolean function on $G F(2)^{t}$ and $\phi$ any mapping from $G F(2)^{t}$ to $G F(2)^{s}$;

$f$ is defined on $G F(2)^{n}$ by:

$$
f(x, y)=x \cdot \phi(y)+g(y), x \in G F(2)^{s}, y \in G F(2)^{t}
$$

where "." is the usual inner product on $G F(2)^{s}$.

Kurosawa and Satoh use this construction in [16] to obtain nonquadratic $P C(\ell)$ of order $k$ functions. They consider linear mappings $\phi$ only. We believe this is a weakness: the nonquadraticity of their function $f$ comes from the fact that the function $g$ involved in its definition is nonquadratic. But the fact that $f$ satisfies $P C(\ell)$ of order $k$ is independent of the choice of $g$. Thus, it must be possible to find an attack on a cryptosystem using such a function, by using this peculiarity.

We study the Maiorana-McFarland functions $f(x, y)=x \cdot \phi(y)+g(y)$ where $\phi$ is not necessarily linear. This wider framework is more difficult to study. But, using a deep result on the dual distance of nonlinear codes due to Delsarte, we show how to obtain such functions satisfying $P C(\ell)$ of order $k$. Moreover, because we get rid of the linearity of $\phi$, these functions can easily be resilient (independently of the choice of $g$ ). They also can have high nonlinearity.

\subsection{Characterization of those Maiorana-McFarland functions which satisfy $P C(\ell)$ of order $k$}

We begin with the criterion $P C(\ell)$. For every $y \in G F(2)^{t}$ we write $\phi(y)=$ $\left(\phi_{1}(y), \cdots, \phi_{s}(y)\right)$, where the $\phi_{i}$ 's (the coordinate functions of $\left.\phi\right)$ are Boolean functions. 
Proposition 8. Let $f$ be a Maurana-McFurland function $f(x, y)=x \cdot \phi(y)+$ $g(y)$ such that:

1. the sum of at least 1 and at most $\ell$ of the coordinates of $\phi$ is balanced;

2. for every nonzero $b \in G F(2)^{t}$ of weight smaller than or equal to $\ell$, and any $y \in G F(2)^{t}$ :

$$
\phi(y+b) \neq \phi(y) .
$$

Then $f$ satisfies $P C(\ell)$.

\section{Proof:}

For every $a \in G F(2)^{s}$ and every $b \in G F(2)^{t}$, we have:

$$
f(x, y)+f(x+a, y+b)=x \cdot[\phi(y)+\phi(y+b)]+a \cdot \phi(y+b)+g(y)+g(y+b) .
$$

If $b=0$ and $1 \leq w(a) \leq \ell$, then because of condition 1 , the function $f(x, y)+$ $f(x+a, y+b)=a \cdot \phi(y)$ is balanced

If $1 \leq w(b) \leq \ell$, then, for every $y$, the function $x \rightarrow f(x, y)+f(x+a, y+b)$ is an affine function and is non-constant thanks to condition 2 ; it is therefore balanced. Thus, if $1 \leq w(a)+w(b) \leq \ell$, the function $f(x, y)+f(x+a, y+b)$ is balanced.

We study now the criterion $P C(\ell)$ of order $k$. Any of the restrictions of a Maiorana-McFarland function $f(x, y)=x \cdot \phi(y)+g(y)$ obtained by keeping constant some coordinates of $x$ and/or of $y$ is a Maiorana-McFarland function. For instance:

- when we keep constant some coordinates of $x$, say $x_{i}=a_{i}, i \in I$, we obtain a Maiorana-McFarland function $x^{\prime} \cdot \phi^{\prime}(y)+g^{\prime}(y)$, where $x^{\prime} \in G F(2)^{n-|I|}$, whose associated mapping $\phi^{\prime}$ can be obtained from $\phi$ by deleting the corresponding coordinates $\phi_{i}, i \in I$ and whose associated function $g^{\prime}$ is equal to $g+\sum_{i \in I} a_{i} \phi_{i}$; - when we keep constant some coordinates of $y$, we obtain a Maiorana-McFarland function $x \cdot \phi^{\prime \prime}\left(y^{\prime}\right)+g^{\prime \prime}\left(y^{\prime}\right)$ whose associated mapping $\phi^{\prime \prime}$ and function $g^{\prime \prime}$ can be obtained from $\phi$ and $g$ by keeping constant the corresponding coordinates of $y$.

Corollary 9. Let $f$ be a Maiorana-McFurland function $f(x, y)=x \cdot \phi(y)+g(y)$ such that:

1. the sum of at least 1 and at most $\ell$ coordinates of $\phi$ is $k$-resilient;

2. if $b \in G F(2)^{t}$ is nonzero and has weight smaller than or equal to $\ell$, then, for every $y \in G F(2)^{t}$, at least $k+1$ coordnuates of the words $\phi(y+b)$ and $\phi(y)$ differ; then $f$ satisfies the propagation craternon $P C(\ell)$ of order $k$.

Proof:

Let $f^{\prime}\left(x^{\prime}, y^{\prime}\right)=x^{\prime} \cdot \phi^{\prime}\left(y^{\prime}\right)+g^{\prime}\left(y^{\prime}\right)$ be the restriction of $f$ obtained by keeping constant at most $k$ coordinates of $x$ and/or $y$. The sum of at least one and at most $\ell$ coordinates of $\phi^{\prime}$ is equal to the restriction of the sum of at least one and at most $\ell$ coordinates of $\phi$. Thus, according to condition 1 of corollary 9 , condition 1 of proposition 8 is satisfied by $f^{\prime}$. According to condition 2 of corollary 9 , for every $y^{\prime}$ and every nonzero $b^{\prime}$ of weight smaller than or equal to $\ell$, the words $\phi^{\prime}\left(y^{\prime}+b^{\prime}\right)$ and $\phi^{\prime}\left(y^{\prime}\right)$ are different from each other and condition 2 of proposition 8 is satisfied by $f^{\prime}$. 
We consider now the mappings of the form $\phi_{2} \circ \phi_{1}$, where $\phi_{1}$ is a mapping from $G F(2)^{t}$ to $G F(2)^{r}$ and $\phi_{2}$ a mapping from $G F(2)^{r}$ to $G F(2)^{s}$. This allows us to split the conditions of corollary 9 into several simpler ones.

Proposition 10. A sufficient condition for a mappang of the form $\phi_{2} \circ \phi_{1}$ to satisfy conditions 1 and 2 of corollary 9 is that:

1. (a) The mapping $\phi_{1}$ is k-resilient;

(b) if two words $y$ and $z$ are different from each other and are at distance smaller than or equal to $\ell$ one to each other, then $\phi_{1}(y) \neq \phi_{1}(z)$;

2. (a) the sum of at least one and at most $\ell$ coordinates of $\phi_{2}$ is balanced;

(b) if $y \neq z$, then $\phi_{2}(y)$ and $\phi_{2}(z)$ differ in at least $k+1$ coordinates.

Proof:

Condition 1 of corollary 9 is satisfied thanks to conditions 1.(a) and 2.(a) (according to property 1 ). Condition 2 is clearly satisfied thanks to the two others.

Kurosawa and Satoh [16] do not study the Maiorana-McFarland functions in general, but their particular construction respects these conditions.

\subsection{Using nonlinear codes}

We want now to design nonlinear mappings $\phi_{1}$ and $\phi_{2}$ satisfying the conditions of proposition 10. The key-notion will be that of dual distance of a (nonlinear) code:

Definition 11. Let $C$ be a nonlinear code of length $n$ (i.e. a subset of $G F(2)^{n}$ ). The distance enumerator of $C$ is the following polynomial in two variables:

$$
D_{C}(X, Y)=\frac{1}{|C|} \sum_{x, y \in C} X^{n-l(1, y)} Y^{l(x, y)}
$$

where $d(x, y)$ is the Hamming distance between the words $x$ and $y$.

The dual distance of $C$ is the smallest positive integer $i$ such that the coefficient of the monomial $X^{n-\imath} Y^{i}$ in the polynomial $D_{C}(X+Y, X-Y)$ is nonzero.

When the code is linear, the dual distance is equal to the minimum distance of the dual code, thanks to the Mac Williams identity (cf. [21]). Even when the code is nonlinear, the dual distance plays an important role, thanks to a result due to Delsarte [9], which can be staterl as follows:

Proposition 12. Let $C$ be a code of dual distance $d^{\perp}$. The indicator of $C$ (i.e. the Boolean function whose support is $\left.C^{\prime}\right)$ is $a\left(d^{\perp}-1\right)$-th order correlation immune function.

We deduce the following two corollaries: 
Corollary 13. Let $C$ be a code of length $n$ and dual distance $d^{\perp}$. Then, for every set $I \subset\{1, \cdots, n\}$ such that $1 \leq|I| \leq d^{\perp}-1$, the function $\sum_{i \in I} x_{i}$ is balanced on C.

This is a direct consequence of proposition 12 and lemma 6.

Corollary 14. Let $\phi$ be a mapping from $G F(2)^{t}$ to $G F(2)^{r}$. If all the codes $\phi^{-1}(c) ; c \in G F(2)^{r}$ have the same cardinality and dual distances greater than or equal to $k+1$, then $\phi_{1}$ is $k$-resilient.

This is a consequence of proposition 12 and of the remark in subsection 4.1 . From proposition 10 and corollaries 13 and 14, we deduce:

Proposition 15. Let $\phi$ be a mapping of the form $\phi=\phi_{2} \circ \phi_{1}$ such that:

1. (a) the codes $\phi_{1}^{-1}(c) ; c \in G F(2)^{r}$ have the same cardinality and dual distances greater than or equal to $k+1$;

(b) these same codes have minimum distances greater than or equal to $\ell+1$;

2. $\phi_{2}$ is injective and:

(a) the code $\phi_{2}\left(G F(2)^{r}\right)$ has dual distance greater than or equal to $\ell+1$;

(b) this same code has nunumum dastance greater than or equal to $k+1$.

Then $\phi$ fulfills the condations of corollary 9 .

We carry on the simplification of the conditions by considering now systematic codes (cf. [21]; see also [23]).

Definition 16. A binary code $C$ of length $n$ is called systematic if there exists a subset $I$ of $\{1, \cdots, n\}$ called an information set of $C$, such that every possible tuple occurs in exactly one codeword within the specified coordinates $x_{i} ; i \in I$.

Denoting by $E_{I}$ the vector-space $\left\{e \in G F(2)^{n} \mid \forall i \in I, e_{i}=0\right\}$, the set of all the cosets $C+e, e \in E_{I}$ is then a partition of $G F(2)^{n}$. Let $\phi_{1}$ be the mapping $x \rightarrow e \in E_{I} \mid x \in C+e$. Then all the codes $\phi_{1}^{-1}(e)=C+e, e \in E_{I}$ have the same cardinality, the same distance enumerator and therefore the same minimum distance and the same dual distance.

Theorem 17. Let $C_{1}$ be a systematic code of length $t$. Let $I=\{r+1, \cdots, t\}$ be an information set of this code.

Let $\phi_{1}$ be the mapping $y \in G F(2)^{t} \rightarrow e \in G F(2)^{r} \mid y \in C_{1}+\left(e_{1}, \cdots, e_{r}, 0, \cdots, 0\right)$. Let $\phi_{2}$ be an injective nupping from $G F(2)^{r}$ to $G F(2)^{s}$ (s>r) and $C_{2}$ the code equal to $\phi_{2}\left(G F(2)^{r}\right)$. Assume that:

1. $C_{1}$ has minimum distance greater than or equal to $\ell+1$ and dual distance greater than or equal to $k+1$;

2. $C_{2}$ has dual distance greater than or equal to $\ell+1$ and minimum distance greater than or equal to $k+1$; 
then the function $f(x, y)=x \cdot\left(\phi_{2} \circ \phi_{1}\right)(y)+y(y), x \in G F(2)^{s}, y \in G F(2)^{t}$, where "." denotes the usual inner product in $G F(2)^{s}$, satisfies $P C(\ell)$ of order $k$.

Every linear code is systematic (cf. [21]). Thus, this result includes that of Kurosawa and Satoh [16].

There exist several known (classes of) nonlinear systematic codes. The two most famous are the $\left(2^{m}, 2^{2 m}, 2^{m-1}-2^{\frac{m}{2}-1}\right.$ ) Kerdock code ( $m$ even $\geq 4$; we give here the length, the cardinality and the minimum distance) whose dual distance is 6 and the $\left(2^{m}, 2^{2^{m}-2 m}, 6\right)$ Preparata code, whose dual distance is $2^{m-1}-2^{\frac{m}{2}-1}$. A class of codes with the same parameters as the Preparata codes has also been recently introluced [11]. All these codes happen to have strictly better parameters than linear codes: for instance, it is proved in [2] that the Preparata code has at least twice more codewords than any linear code with the same length and the same minimum distance. It is also proved that the Kerdock code has more codewords than any linear code with the same length and the same dual distance.

Corollary 18. For any even $m \geq 4$, there exists a mapping $\phi: G F(2)^{2^{m}} \rightarrow$ $G F(2)^{2^{m}}$ such that, for any Boolean function g on $G F(2)^{2^{m}}$, the function $f(x, y)=$ $x \cdot \phi(y)+g(y)$ is a nonquadratic Boolean function on $G F(2)^{2^{m+1}}$ satisfying $P C(5)$ of order $2^{m-1}-2^{\frac{m}{2}-1}-1$ (resp. $P C\left(2^{m-1}-2^{\frac{m}{2}-1}-1\right)$ of order 5$)$.

Other examples of nonlinear systematic codes are the $\left(2^{m}, 2^{2 m+k(m-1)}, 2^{m-1}-\right.$ $2^{\frac{m}{2}+k-1}$ ) Delsarte-Goethals codes ( $m$ even, $k<\frac{m}{2}$ ) and their formal duals (cf. [12]).

\subsection{Resilience and nonlinearity of the functions}

The propagation criterion of degree $\ell$ and order $k$ is not sufficient for a cryptographic function. The function needs also to be balanced - or, better, to be resilient of a sufficient order - and to have high nonlinearity (i.e. distance to the set of all affine functions).

The resilience order of the Maiorana-McFarland functions has been determined:

Proposition 19. (5) Let $f$ be a Maioranu-McFurland function:

$$
f(x, y)=x \cdot \phi(y)+g(y), x \in G F(2)^{s}, y \in G F(2)^{t}
$$

where $\phi$ is a mapping from $G F(2)^{t}$ to $G F(2)^{s}$ and $g$ a Boolean function on $G F(2)^{t}$. Function $f$ is $\tau$-resilkent if and only if every word of the set $\phi\left(G F(2)^{t}\right)$ has weight greater than or equal to $\tau+1$.

Thus, the function $f$ designed in theorem 17 is $(w-1)$-resilient, where $w$ is the minimum weight of all the words in $C_{2}$. Thus, the greatest resilience order which can be achieved for such a function is equal to the covering radius of the code $C_{2}$ (cf. [21]). For instance, the covering radius of the Kerdock code (resp. the 
Preparata code) being greater than or equal to $2^{m-1}-2^{\frac{m}{2}}$ (resp. being equal to 3 ), the function $f$ of corollary 18 can be defined so that it is $\left(2^{m-1}-2^{\frac{m}{2}}-1\right)$ resilient (resp. 2-resilient).

It is also a simple matter to obtain functions with high nonlinearity.

\section{References}

1. J. Bierbrauer, K. Gopalakrishnan and D.R. Stinson. Bounds for resilient functions and orthogonal arrays. Advances in Cryptology, CRYPTO'94, Lecture Notes in Cornputer Sciences, Springer Verlag $\mathrm{n}^{\circ} 839$, pages 247-256 (1994)

2. A.E. Brouwer and L.M.G.M. Tholhuizen. A sharpening of the Johnson bound for binary linear codes and the nonexistence of linear codes with Preparata parameters Designs, Codes and Cryptography 3, pages 95-98 (1993)

3. P. Camion and A. Canteaut. Construction of $t$-resilient functions over a finite alphabet, Advances in C'ryptrology, EUROCRYPT'96, Lecture Notes in Computer Sciences, Springer Verlag ${ }^{\circ}$ 1070, pages 283-293 (1996)

4. P. Camion and A. Canteaut. Generalization of Siegenthaler inequality and Schnorr-Vaudenay multipermutations. In N. Koblitz, editor, Advances in Cryptology - CRYPTO'96, number 1109 in Lecture Notes in Computer Science, pages 372-386 Springer-Verlag, 1996.

5. P. Camion, C. Carlet, P. Charpin and N. Sendrier. On correlation-immune functions. Advances an Cryptology, CRYPTO'91, Lecture Notes in Computer Sciences, Springer Verlag $\mathbf{n}^{\circ}$ 576, pages 86-100 (1992)

6. C. Carlet. Hyperbent functions. PRAGOCRYPT'96, Czech Technical University Publishing House, pages 145-155 (1996).

7. C. Carlet. More correlation-immune and resilient functions over Galois fields and Galois rings. Advances in Cryptology, EUROCRYPT' 97, Lecture Notes in Computer Science 1233, pages 422-433, Springer Verlag (1997)

8. P. Delsarte. An algebraic approach to the association schemes of coding theory. Thesis, Université Catholique de Louvain (1973).

9. P. Delsarte. Four fundamental parameters of a code and their combinatorial significance. Information and Control, vol 23, n² 5, pages 407-438 (1973).

10. J. F. Dillon. Elementary Hadamard Difference sets. Ph. D. Thesis, Univ. of Maryland (1974).

11. A. R. Hammons Jr., P. V. Kumar, A. R. C'alclerbank, N. J. A. Sloane and P. Solé. The $Z_{4}$-linearity of Kerdock, Preparata, Goethals and related codes. IEEE Transactions on Information Theory, vol 40, pages 301-320, (1994)

12. F.B. Hergert. On the Delsarte-Goethals codes and their formal duals. Discrete Math. 83, pages 249-263 (1990)

13. S. Hirose and K. Ikeda. Nonlinearity criteria of Boolean functions. KUIS Technical Report, KUIS-94-0002 (1994); postcript file on the web page:

http://www.lab1.kuis.kyoto-u.ac.jp/members/hirose/publications-e.html

14. S. Hirose and $K$. Ikeda. Complexity of Boolean functions satisfying the propagation criterion. The Proc. of the 1995 Symposium on Cryptography and Information Security, SCIS95-B3.3, (1995).

15. T. Jakobsen and L.R. Knudsen. The interpolation attack on block ciphers. Preproc. of Fast Softurate Encryption, pages 28-40 (1997). 
16. K. Kurosawa and T. Satoh. Design of $S A C / P C(\ell)$ of order $k$ Boolean functions and three other cryptographic criteria. Advances in Cryptology, EUROCRYPT' 97, Lecture Notes in Computer Science 1233, pages 434-449, Springer Verlag (1997)

17. W. Meier and O. Staffelbach. Nonlinearity Criteria for Cryptographic Functions. Advances in Cryptology, EUROCRYPT' 89, Lecture Notes in Computer Science 434, pages 549-562, Springer Verlag (1990)

18. B. Preneel, W. Van Leekwijck, L. Van Linden, R. Govaerts and J. Vandevalle. Propagation characteristics of Boolean functions, Advances in Cryptology, EUROCRYPT'90, Lecture Notes un Cornputer Sciences, Springer Verlag $\mathbf{n}^{\circ} 473$, pages 161-173 (1991)

19. B. Preneel, R. Govaerts and J. Vandevalle. Boolean functions satisfying higher order propagation criteria, Advances in Cryptology, EUROCRYPT'91, Lecture Notes in Computer Sciences, Springer Verlag n ${ }^{\circ}$ 547, pages 141-152 (1991)

20. O. S. Rothaus. On bent functions. J. Comb. Theory, 20A, pages 300-305(1976)

21. F. J. Mac Williams et N. J. Sloane, The theory of error-correcting codes, Amsterdam, North Holland 1977.

22. D.R. Stinson. Resilient functions and large sets of orthogonal arrays. Congressus Numer., vol 92, pages $105-110$ (1993)

23. D.R. Stinson and J.L. Massey. An infinite class of counterexamples to a conjecture concerning nonlinear resilient functions. Joutrual of Cryptology, vol $8, \mathrm{n}^{\circ} 3$, pages 167-173 (1995)

24. A. Webster and S. Tavares. On the design of S-boxes. Advances in Cryptology, CRYPTO'85, Lecture Notes in Computer Science $n^{\circ} 218$, pages 523-534 (1985) 